\title{
Theoretical Studies of Diffusion Kinetics in Austenite
}

\author{
A.V. Evteev1,a, E.V. Levchenko ${ }^{1, b}$, I.V. Belova ${ }^{1, c}$ and G.E. Murch ${ }^{1, d}$ \\ ${ }^{1}$ Diffusion in Solids Group, Centre for Geotechnical and Materials Modelling \\ School of Engineering, The University of Newcastle \\ Callaghan, NSW 2308 Australia
}

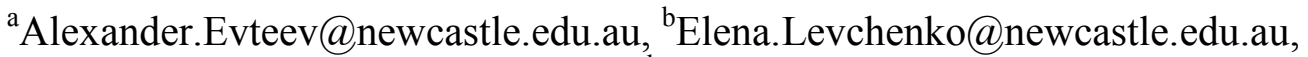 \\ 'Irina.Belova@newcastle.edu.au, dGraeme.Murch@newcastle.edu.au
}

Keywords: diffusion, austenite, computer simulation

\begin{abstract}
We report on the computer simulation (using molecular dynamics and lattice relaxation) to explore tracer and chemical diffusion (carbon) kinetics in austenite at low carbon contents. It was found by molecular dynamics simulations that the detailed balance relations are not valid for the second nearest neighbours in the movements of the carbon interstitial atoms. The effect of a possible split energy level at the second nearest neighbour positions is analysed from a theoretical point of view.
\end{abstract}

\section{Introduction}

Diffusion of iron and carbon in ferrite, austenite and cementite has recently become of interest in order to understand the mechanistic details of metal dusting and also to understand the growth mechanisms of carbon nanotubes by way of metal nanoparticle templates. In this paper, we address carbon diffusion in austenite (the f.c.c. phase). We report on the computer simulation (using molecular dynamics and lattice relaxation) to explore carbon tracer and chemical diffusion kinetics in austenite at low carbon contents.

\section{The model}

We consider an f.c.c. crystal with a very small concentration of interstitial $\mathrm{C}$ atoms. We assume that the concentration of interstitials is low enough that the probability to find three and more neighbouring interstitial atoms can be neglected. Let $w_{0}$ be the frequency with which an interstitial atom moves from one octahedral site to another without interacting with the second interstitial atom. Next, we introduce the frequencies with which any atom from every two interacting interstitial atoms can move: $w_{i 1}, i=1,2,3,4$ are the frequencies with which an atom in a first nn pair moves with the final result being either reorientation of the first nearest neighbour nn pair formation of a $i$-th nn pair; $w_{i 2}, i=1,3,5$ are the dissociative frequencies of the second nn pair with the formation of a $i$-th nn pair; similarly $w_{13}$ and $w_{23}, w_{14}, w_{25}$ are the dissociative frequencies of a third, fourth and fifth nn pairs (according to the second digit in the subscript) with the formation of a first, second nn pairs (according to the first digit in the subscript). All other interstitial jumps in this model are assumed to have the same frequency $w_{0}$ as the jumps of isolated (free) interstitial atoms. The interaction of the interstitial atoms in the third and fourth nn pairs, in contrast to the second nn pairs, through the solvent atoms are quite weak, therefore we will assume that:

$$
w_{31}=w_{41} \text { and } w_{13}=w_{14}
$$

We also assume that the creation of the third, fourth and higher nn pairs occur at random.

In [1] results of an extensive molecular dynamics studies were presented using $\mathrm{Fe}-\mathrm{Fe}$ and $\mathrm{Fe}-\mathrm{C}$ potentials. At the nn position the very weak $\mathrm{C}-\mathrm{C}$ potential is represented only by self-blocking between $\mathrm{C}$ atoms, this affects only $w_{21}, w_{11}$ and $w_{31}$. Then, by making use of the experimental data for the tracer diffusion coefficient, chemical diffusion coefficient and thermodynamic factors and calculating (using Monte Carlo) the tracer correlation factor (most probably there is no collective correlation effect in the chemical diffusion coefficient for this model, in [2] this statement was 
proved for the usual interstitial model) we were able to make the necessary adjustments for these three exchange frequencies.

For $T=1273 K$ the set of the calculated exchange frequencies are (expressed in term of $\left.w_{0}\right)$ [1]:

$$
\begin{aligned}
& w_{11} \approx 0.60 w_{0}, w_{21} \approx 0.36 w_{0}, w_{31} \approx 0.98 w_{0}, w_{12} \approx 0.44 w_{0}, w_{32} \approx 1.68 w_{0}, \\
& w_{52} \approx 1.03 w_{0} ; w_{13} \approx w_{0} ; w_{23} \approx w_{0} ; w_{25} \approx w_{0} .
\end{aligned}
$$

In order to apply exact kinetic theory for the calculation of the tracer correlation factor that is outlined in a series of studies [3-5], we need to check first if these exchange frequencies satisfy the detailed balance relations. First, we let $c_{1}, c_{2}, c_{3}, c_{4}$ and $c_{f}$ be the concentrations of the first, second, third, fourth $\mathrm{nn}$ pairs and the concentration of single atoms respectively at thermal equilibrium and $c_{1}=6 \delta_{1} c_{\mathrm{f}}^{2}, c_{2}=3 \delta_{2} c_{\mathrm{f}}^{2}, c_{3}=12 c_{f}^{2}, c_{4}=6 c_{f}^{2}$, where $\delta_{1}$ and $\delta_{2}$ are deviation of the concentrations $c_{1}$, $c_{2}$ from the case of random creation (obviously $\delta_{i}>1$ corresponds to attraction, $\delta_{i}<1$ corresponds to repulsion between the carbon atoms at $i$-th nn configuration). The detailed balance relations then state that:

$$
\begin{aligned}
& \delta_{1}=w_{13} / w_{31} ; \\
& \delta_{2}=w_{23} / w_{32}=w_{25} / w_{52}=w_{21} / w_{12} \delta_{1} .
\end{aligned}
$$

Substituting data of Eq. 1 into Eqs 3 we can see that there is a problem with $\delta_{2}$. There is a significant discrepancy between three values: $w_{23} / w_{32}=0.595, \quad w_{25} / w_{52}=0.971$ and $w_{21} / w_{12} \delta_{1}=0.367$. This means that the detailed balance relations are no longer satisfied and consequently application of any standard diffusion kinetics theory becomes impossible. Moreover, as was pointed out in [4], application of usual Monte Carlo simulations is no longer valid in such a situation.

To overcome this problem, we will undertake more numerical analysis by molecular dynamics simulations to find the reason for the break of detailed balance at the second nn configurations. There could however be several possible reasons for this effect. The most probable candidate to explain it would be the effect of the energy level at the second nn configuration being effectively split into three local minima (see Figure 1 for the case of 'double' local minima: for jumps from the second to the first nn configuration and reverse and for jumps from the second to the third nn configuration and reverse). This effect is obviously a very subtle one and will require careful analysis of the already obtained numerical simulations data and possibly construction of the very detailed energy landscapes for the second nn configuration. The other possibility could be that the energy paths from the second nn positions are different from the corresponding paths to the second $\mathrm{nn}$ positions. In principle, this scenario would be extremely difficult to cope with. But the high probability that the deviation from the detailed balance relations happens only at the second $\mathrm{nn}$ configuration would be of great help and we would be able to reduce this scenario to the split energy level scenario.

Possible solution: split energy level description The split energy level effect at an atomic site can be introduced in the following way. The split energy effect must be accompanied by 'splitting' of the second nn sites in space. This means that instead of just one position 2 we have 3 different positions $\left(2^{1}, 2^{3}\right.$ and $\left.2^{5}\right)$ located somewhere in the vicinity of the usual atomic site. Then we should assume the following conditions of an atomic jump from these three possible locations: if the atom resides in location $2^{1}$ it can only jump into the first nearest neighbour sites, if the atom resides in location $2^{3}$ it can only jump into the third nearest neighbour sites, if the atom resides in location $2^{5}$ it can only jump into the fifth nearest neighbour sites (see Figure 2).

Now we can introduce probabilities of finding carbon atoms at the positions $2^{1}, 2^{3}$ and $2^{5}$ :

$$
c_{2}^{1}=3 \delta_{2}^{1} c_{\mathrm{f}}^{2}, \quad c_{2}^{3}=3 \delta_{2}^{3} c_{\mathrm{f}}^{2}, \quad c_{2}^{5}=3 \delta_{2}^{5} c_{\mathrm{f}}^{2}
$$




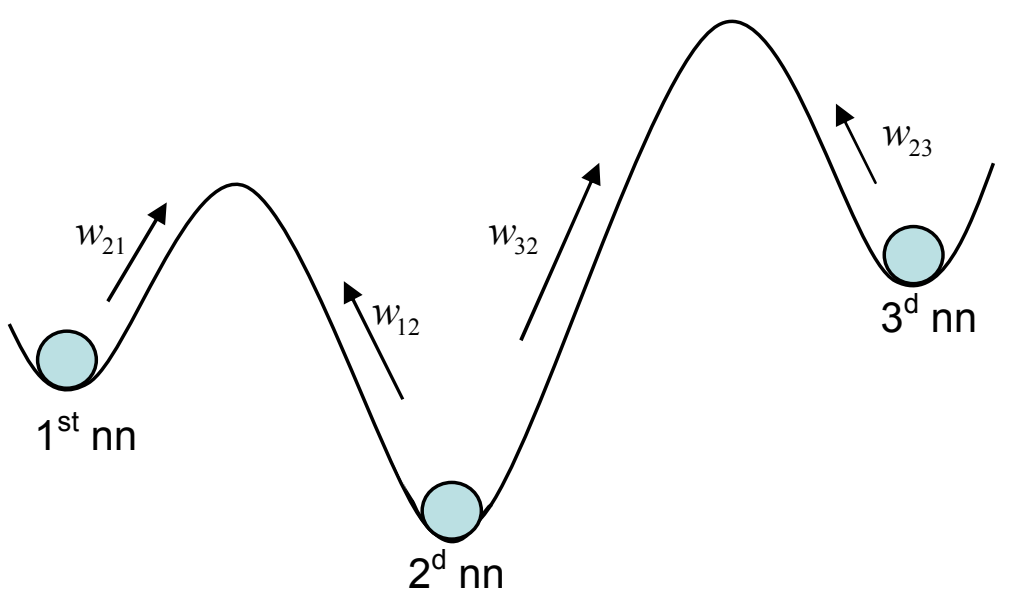

(a)

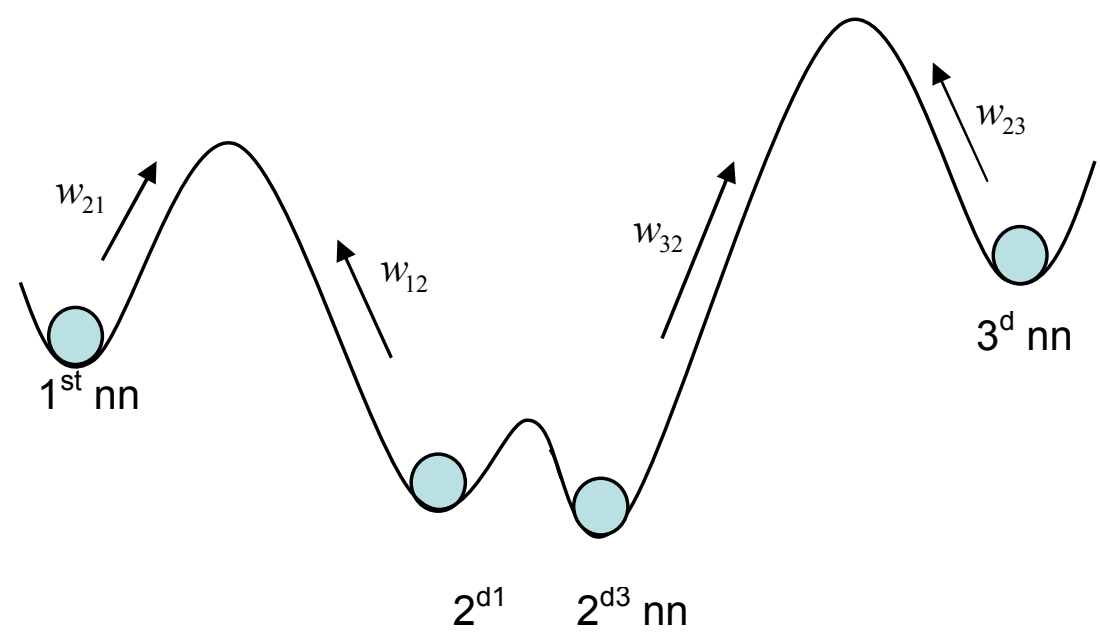

(b)

Figure 1. (a) - Conventional description of the energy level between neighbouring sites; (b) modification required for the case of carbon interstitial diffusion: at the second nn configuration the split energy level occurs.

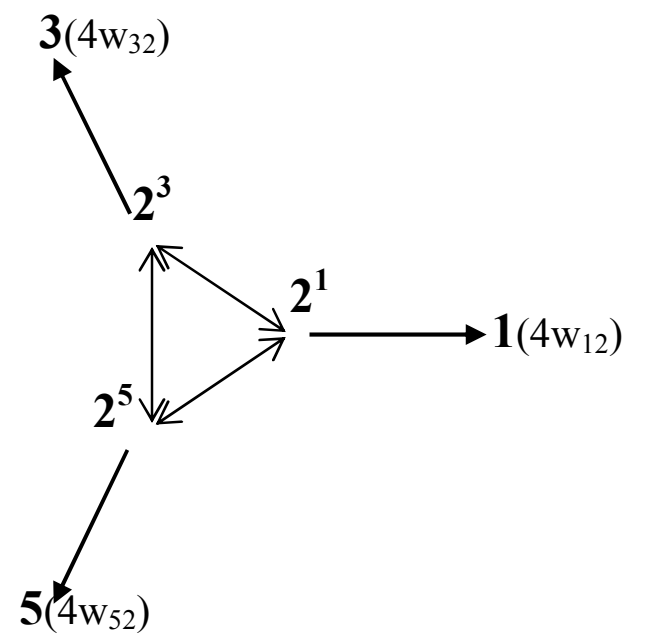

Figure 2. Schematic presentation of the split energy level at the second nn configuration.

And then it is easy to see that: 


$$
\delta_{2}^{1}=w_{13} w_{21} /\left(w_{31} w_{12}\right), \quad \delta_{2}^{3}=w_{23} / w_{32}, \quad \delta_{2}^{5}=w_{25} / w_{52}
$$

where $\delta_{2}{ }^{1}, \delta_{2}{ }^{3}$ and $\delta_{2}{ }^{5}$ are the deviations of the concentrations from the corresponding concentrations occurring at random. The total concentration of the first four nn interstitial pairs is:

$$
c_{\mathrm{p}}=c_{1}+c_{2}+12 c_{f}^{2}+6 c_{f}^{2}=27 \delta c_{\mathrm{f}}^{2}, \quad \delta=\left(2 \delta_{1}+\delta_{2}+6\right) / 9
$$

where we have used the relation: $c_{2}=\left(c_{2}^{1}+c_{2}^{3}+c_{2}^{5}\right) / 3$ and accordingly $\delta_{2}=\left(\delta_{2}^{1}+\delta_{2}^{3}+\delta_{2}^{5}\right) / 3$. The factor $1 / 3$ here reflects the condition that each of the positions $2^{1}, 2^{3}$ and $2^{5}$ 'responsible' for 4 coordinations out of the total 12 (Figure 2).

The total concentration of the interstitial atoms is:

$$
c=c_{\mathrm{f}}+2 c_{\mathrm{p}}=c_{\mathrm{f}}\left(1+54 \delta c_{\mathrm{f}}\right)
$$

The average number of jumps $\Gamma$ made by an interstitial atom per unit time at thermal equilibrium can then be found by simply averaging over all possible types of jumps:

$$
c \Gamma=2 c_{1} \Gamma_{1}+2 c_{2} \Gamma_{2}+2 c_{3} \Gamma_{3}+2 c_{4} \Gamma_{4}+24 c_{f}^{2} \Gamma_{5}+\left(c_{f}-24 c_{f}^{2}\right) \Gamma_{0},
$$

Where

$$
\begin{gathered}
\Gamma_{0}=12 w_{0}, \quad \Gamma_{1}=4 w_{11}+2 w_{21}+5 w_{31}, \quad \Gamma_{2}=4\left(w_{12} \delta_{2}^{1}+w_{32} \delta_{2}^{3}+w_{52} \delta_{2}^{5}\right) / \delta_{2}, \\
\Gamma_{3}=2 w_{13}+w_{23}+9 w_{0}, \quad \Gamma_{4}=w_{13}+11 w_{0}, \quad \Gamma_{5}=w_{25}+11 w_{0}
\end{gathered}
$$

are respectively the average number of jumps made by the free interstitial atoms, and by atoms at the first, second, third, fourth and fifth nn interstitial pairs per unit time. To first order in the total interstitial concentration (see Eq. 7) we will then have that:

where

$$
\Gamma=\Gamma_{0}\left(1+\varepsilon_{\Gamma} c\right)
$$

$$
\begin{aligned}
\varepsilon_{\Gamma}= & {\left[\delta_{1}\left(4 w_{11}+2 w_{21}+5 w_{31}-12 w_{0}\right)+2\left(w_{12} \delta_{2}^{1}+w_{32} \delta_{2}^{3}+w_{52} \delta_{2}^{5}-3 \delta_{2} w_{0}\right)+\right.} \\
& \left.+2 \delta_{3}\left(2 w_{13}+w_{23}-3 w_{0}\right)+\delta_{4}\left(w_{13}-w_{0}\right)+2\left(w_{25}-w_{0}\right)\right] / w_{0} .
\end{aligned}
$$

For the chemical diffusion coefficient of interstitial atoms we can write to first order in the total interstitial concentration:

$$
\widetilde{D}=b^{2} \Gamma \alpha / 3=b^{2} \Gamma_{0}\left(1+\varepsilon_{\widetilde{D}} c\right) / 3,
$$

where $\sqrt{ } 2 b$ is the nn separation, $\varepsilon_{\widetilde{D}}=\varepsilon_{\Gamma}+\varepsilon_{\alpha}, \varepsilon_{\Gamma}$ is the contribution from the average number of jumps $\Gamma$ made by an interstitial atom per unit time, $\varepsilon_{\alpha}$ is the contribution from the thermodynamic factor $\alpha\left(\alpha=1+\varepsilon_{\alpha} c\right)$ into $\varepsilon_{\widetilde{D}}$.

In [1] it was shown that $\varepsilon_{\widetilde{D}}$ should be given by the following expression:

$$
\begin{aligned}
& \varepsilon_{\widetilde{D}}=2\left[2 \delta_{1}\left(w_{11}+w_{21}+4 w_{31}-6 w_{0}\right)+2\left(w_{32} \delta_{2}^{3}+2 w_{52} \delta_{2}^{5}-3 \delta_{1} w_{0}\right)-\right. \\
& \left.-\left(2 \delta_{3}+\delta_{4}\right)\left(w_{13}-w_{0}\right)-2\left(w_{25}-w_{0}\right)\right] / w_{0}
\end{aligned}
$$

Here, we have taken into account the 'effective' split energy level at the second nn configuration. 
Similarly, for the tracer diffusion coefficient of the carbon interstitial atoms we can write to first order in the total interstitial concentration:

$$
D^{*}=b^{2} \Gamma f / 3=b^{2} \Gamma_{0}\left(1+\left(\varepsilon_{\Gamma}+\varepsilon_{f}\right) c\right) / 3,
$$

The experimental data at $1273 \mathrm{~K}$ are $\varepsilon_{\widetilde{D}}^{\exp } \approx 8.44$ [1] and for the $\varepsilon_{\alpha}^{\exp }$ we accept the upper bound as $\varepsilon_{\alpha}^{\exp (1)} \approx 7.69$ given in [6], and the lower bound $\varepsilon_{\alpha}^{\exp (2)} \approx 5.68$ [7]. In [1], in order to adjust $w_{11}, w_{21}$ and $w_{31}$ (and to be able to use experimental tracer diffusion data) we used tracer correlation factors that were calculated by Monte Carlo simulation. But for the split energy level at the second nn configuration conventional Monte Carlo simulation is not appropriate. A generalization of the kinetic theory for the tracer correlation factor is in the process and will be published elsewhere.

An approximate averaging procedure of the split energy level can be also used. If we average the exchange frequencies according to the required detailed balance then it can be easily shown that we still can have a conventional description of the jumps from the second position with effective exchange frequencies instead of the measured ones (see Figure 3).

Effective exchange frequencies are different from the measured ones by factors coming about from the differences between the energy levels on the $2^{1}, 2^{3}$ and $2^{5}$ locations.

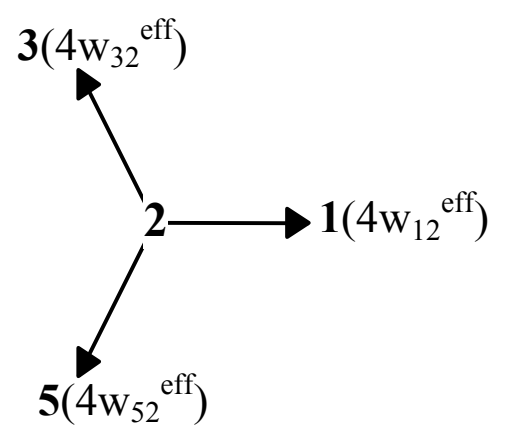

Figure 3. Schematic presentation of the result of the averaging procedure of the 'partial' concentrations of the second nn configuration.

Using Eqs 4,5 we soon have

$$
c_{2}^{1}=\frac{3 \delta_{2}^{1}}{\delta_{2}^{1}+\delta_{2}^{3}+\delta_{2}^{5}} c_{2} ; \quad c_{2}^{3}=\frac{3 \delta_{2}^{3}}{\delta_{2}^{1}+\delta_{2}^{3}+\delta_{2}^{5}} c_{2} ; \quad c_{2}^{5}=\frac{3 \delta_{2}^{5}}{\delta_{2}^{1}+\delta_{2}^{3}+\delta_{2}^{5}} c_{2}
$$

Then the required effective (averaged) exchange frequencies are:

$$
w_{12}^{e f f}=\frac{3 \delta_{2}^{1}}{\delta_{2}^{1}+\delta_{2}^{3}+\delta_{2}^{5}} w_{12} ; \quad w_{32}^{\text {eff }}=\frac{3 \delta_{2}^{3}}{\delta_{2}^{1}+\delta_{2}^{3}+\delta_{2}^{5}} w_{32} ; \quad w_{52}^{\text {eff }}=\frac{3 \delta_{2}^{5}}{\delta_{2}^{1}+\delta_{2}^{3}+\delta_{2}^{5}} w_{52}
$$

We should note first that this approach is somewhat approximate. Secondly, if there is energy splitting at the first nn positions as well, then we will not be able to solve the system of detailed balance relations. In that case, additional information will be required.

\section{Application to the carbon interstitial diffusion MD calculations at 1273K}

We can apply relations Eqs 16 to have $w_{12}{ }^{\text {eff }}=0.458 ; w_{32}{ }^{\text {eff }}=1.251=w_{52}{ }^{\text {eff }}$. With these corrections we can use Monte Carlo simulation results for the tracer correlation factors (or tracer correlation 
theory). The final results of the corrected exchange frequencies are similar to those calculated in [1]:

$$
w_{11}^{\text {corr }} \approx 1.01 w_{0}, w_{21}^{\text {corr }} \approx 0.64 w_{0}, w_{31}^{\text {corr }} \approx 1.75 w_{0}, w_{12} \approx 0.44 w_{0}, w_{32} \approx 1.68 w_{0}, w_{52} \approx 1.03 w_{0}
$$

With these corrected set we have $\varepsilon_{\alpha}^{\text {exp }}=7.0$.

\section{Summary}

We reported on the computer simulation (using molecular dynamics and lattice relaxation) to explore tracer and chemical diffusion (carbon) kinetics in austenite at low carbon contents. It was found by molecular dynamics simulations that the detailed balance relations were not valid for the second nearest neighbours in the movements of the carbon interstitial atoms. The effect of a possible split energy level at the second nearest neighbour positions was analysed from a theoretical point of view.

\section{Acknowledgement}

We are very grateful for the fruitful discussions and support of Prof A.R. Allnatt (University of Western Ontario, Canada). We wish to thank the Australian Research Council for its support of this work.

\section{References}

[1] A.V. Evteev, E.V. Levchenko, I.V. Belova and G.E. Murch: Phil. Mag., to be published. [2] I.V. Belova, M.J. Brown, A.R. Allnatt and G.E. Murch: Phil. Mag. Vol. 85 (2005), p. 2787.

[3] A.R. Allnatt, Diffusion in Materials, edited by A.L. Laskar, J.L.Bocquet, G. Brebec and C. Monty (Kluwer Academic Publishers, Dordrecht/Boston/London 1990).

[4] A.R. Allnatt and A.B. Lidiard, Atomic Transport in Solids, (University Press, Cambridge 1993).

[5] M. Koiwa and S. Ishioka: Phil. Mag. Vol. A47 (1983), p. 927.

[6] S. Ban-ya, J.F. Elliott and J. Chipman: J. Metall. Trans. Vol. 1 (1970), p. 1313.

[7] H. Aaronson, H.A. Domian and G.M. Pound: Trans TMS-AIME Vol. 236 (1966), p. 753. 